\title{
Politische Ökologie des Postsozialismus
}

\author{
Matthias Schmidt \\ Institut für Geographie, Universität Augsburg, Augsburg, Germany \\ Correspondence to: Matthias Schmidt (schmidt@geo.uni-augsburg.de)
}

Received: 7 March 2016 - Revised: 4 July 2016 - Accepted: 7 July 2016 - Published: 17 October 2016

\begin{abstract}
Kurzfassung. The consequences and effects of the socialist experiment are still felt today, a quarter century after the system change in Eastern Europe and the Soviet Union. The political, economic and social transformation processes which occurred in this period have only partially led to a convergence with the West. The former socialist societies show, to this day, striking differences and specific paths, which is why "postsocialism" as an explanatory category is still of relevance. Although political ecology was established decades ago, its research activities touching various fields and resulting in numerous studies conducted in rural and urban contexts around the world, there are hardly any political-ecological studies that deal with the post-socialist reality of Eurasia. A central field of political ecology stems from questions about the relationships between political and socioeconomic transformation processes, on the one hand, and poverty, vulnerability and environmental change, on the other. Such problems occur in a particularly striking manner in the former "Second World" countries. Here, the spatial and temporal multiscalar approach of political ecology appears well suited to analyse the transformations of human-environment relationships. Political-ecological studies in the post-socialist sphere must take into account some special features, such as the legacy of socialist modernization, the disastrous environmental damages of the planned economy or the reconfiguration of institutions. This article discusses the conceptual particularities and challenges for a political ecology of post-socialism.
\end{abstract}

\section{Einleitung}

Das Ende des Kalten Krieges, der Niedergang der osteuropäischen Planwirtschaften und der Kollaps der Sowjetunion in den Wendejahren 1989/91 markieren unzweifelhaft eine epochale historische Wende globaler Dimension. Abgesehen von den weltpolitischen Erschütterungen setzten in den betroffenen Gesellschaften des ehemals ,real existierenden Sozialismus" umfassende Transformationsprozesse ein. Denn der politische Systemwechsel wurde begleitet von massiven ökonomischen und soziokulturellen Transformationen, die sich auch auf das Verhältnis von Mensch und Natur bzw. Gesellschaft und Umwelt auswirkten.

Die Vereinten Nationen und die Weltbank führten die Staaten der ehemaligen „Zweiten Welt" fortan als Countries in Transition (World Bank, 1996), da sie bestimmte Partikularitäten aufweisen, die sie von westlichen Industrieländern und den sogenannten Entwicklungsländern Lateinamerikas, Afrikas oder Asiens deutlich unterschieden. Begleitet war diese Kategorisierung zudem mit der Erwartung - und Hoff- nung -, dass sich in diesen nun postsozialistischen Ländern Demokratie und Marktwirtschaft durchsetzen und über kurz oder lang eine Konvergenz mit den westlichen Gesellschaften einstellen würden. Doch wie verschiedene Indikatoren zu Entwicklung, Wohlstand, Demokratie oder Pressefreiheit demonstrieren (Bertelsmann Stiftung, 2016; UNDP, 2015; Freedom House, 2015), die politischen Zielrichtungen gegenwärtiger Regierungen wie beispielsweise in Weißrussland, Aserbaidschan oder Usbekistan verdeutlichen und auch aktuelle Umfragen zur Attraktivität des westlichen Modells zeigen (Russische Akademie der Wissenschaften, 2016), ist eine solche Konvergenz nicht eingetreten. Zwar haben in mancherlei Hinsicht die politischen und ökonomischen Entwicklungen der vergangenen 25 Jahre zu einer Angleichung der postsozialistischen Gesellschaften an den „Westen“ geführt, dennoch weisen sie spezifische Entwicklungspfade auf und zeigen bis heute markante Differenzen.

Ausgehend von dieser Prämisse ist es äußerst verwunderlich, dass sich bislang nur eine überschaubare Anzahl explizit politisch-ökologischer Studien mit den Bedingun- 
gen und Veränderungen im ehemaligen „Ostblock“ auseinandersetzen. Dabei böten die so differenten Materialitäten, Strukturen, Akteursgruppen und Prozesse, etwa im Hinblick auf Wahrnehmung und Management von Naturräumen und -ressourcen, Umweltwandel und -degradation, Naturund Umweltschutz, vielerlei Fragestellungen im Forschungsfeld der Politischen Ökologie. Beispielsweise danach, welche Maxime dem Naturschutz in der Sowjetunion zugrunde lag oder welche Widerstände gegen die oftmals rücksichtslose Ressourcenextraktion artikuliert und erfolgreich durchgesetzt wurden. Denkbare Fragen wären auch, wie Raumkategorisierungen, institutionelle Strukturen oder Eingriffe in den Naturhaushalt bis heute als socialist legacies gegenwärtige Entwicklungen beeinflussen. Gerade der räumlich und zeitlich multiskalare Ansatz der Politischen Ökologie erscheint bestens geeignet, die durch politische Systemwechsel geprägten Gesellschaften und Umwelten der postsozialistischen Sphäre zu untersuchen. Dies allerdings findet kaum statt.

Vor diesem Hintergrund verfolgt der vorliegende Aufsatz das Ziel, einen Beitrag zur Etablierung einer Politischen Ökologie des Postsozialismus zu leisten, mindestens jedoch die Politische Ökologie um eine Perspektive auf postsozialistische Gesellschaft-Umwelt-Verhältnisse zu erweitern. Nach einem kurzen Abriss zum Stand der Politischen Ökologie, ihren Schwerpunkten und Unzulänglichkeiten werden Gründe einer Beschäftigung der Politischen Ökologie mit der postsozialistischen Welt aufgezeigt, Besonderheiten postsozialistischer Gesellschaft-Umwelt-Verhältnisse erörtert und Prinzipien einer wissenschaftlichen Auseinandersetzung der Politischen Ökologie mit postsozialistischen Gesellschaften und Umwelten skizziert.

\section{Schwerpunkte der Politischen Ökologie und eine Leerstelle}

Die Politische Ökologie entwickelte und etablierte sich in den vergangenen Dekaden zu einer anerkannten und einflussreichen Forschungsrichtung innerhalb der Geographie, der Ethnologie und weiteren Sozialwissenschaften. Insbesondere im angloamerikanischen Raum ist sie äußerst populär und fand Eingang in zahlreiche Curricula Geographischer Institute. Die Gründung der Zeitschrift Journal of Political Ecology, die mitgliederstarke Cultural and Political Ecology Specialty Group of the Association of American Geographers (CAPE-AAG) sowie ungezählte wissenschaftliche $\mathrm{Pu}$ blikationen zeugen von einer großen Rezeption und bis heute anhaltenden Vitalität der Politischen Ökologie. ${ }^{1}$

\footnotetext{
${ }^{1}$ Auch im deutschsprachigen Raum verfügt die Politische Ökologie über eine nicht unbeträchtliche Popularität, wurde in Themenheften der Zeitschrift für Wirtschaftsgeographie und der Geographischen Rundschau sowie in verschiedenen Publikationen (z.B., Krings und Müller, 2001; Schneider, 2002; Soliva, 2002, Flitner, 2007; Krings, 2008, 2011) ausführlich diskutiert (siehe Beitrag von
}

In den gemeinhin als die zentralen Gründungswerke geltenden Publikationen von Watts (1983), Blaikie (1985), Blaikie und Brookfield (1987) sowie in vielen folgenden Studien (z.B. Bryant und Bailey, 1997; Zimmerer und Bassett, 2003) standen Umweltdegradation und -wandel sowie Machtverhältnisse und politische Konflikte um Naturressourcen in ländlichen Räumen des Globalen Südens im Fokus der Politischen Ökologie. Die Bandbreite wurde durch Studien im Globalen Norden (z.B. McCarthy, 2002) und in städtischen Räumen (z.B. Swyngedouw und Heynen, 2003) sowie durch historische (Davis, 2009) und globale Perspektiven (Peet et al., 2011) erweitert, fokussierte somit sowohl dünn besiedelte Räume als auch hoch verdichtete und soll schließlich auch die ,in-betweens“ (Bryan et al., 2011) nicht vernachlässigen. Von zentraler Bedeutung ist in vielen Studien der Bezug auf den Zusammenhang von Kapitalismus und Staatsintervention einerseits und Marginalisierung und Umweltdegradation andererseits.

Seit einiger Zeit wird - nicht zu Unrecht - die Fuzziness der Politischen Ökologie beklagt, die als Forschungsagenda (Bryant, 1992), Ansatz (Zimmerer und Bassett, 2003), Perspektive (Rocheleau et al., 1996) oder schlichtweg nur als Betitelung für unterschiedlichste wissenschaftliche Problemund Fragestellungen herhalten muss, da scheinbar alles und jedes unter diesem Banner subsumiert werden kann. Aufgrund ihrer Offenheit bzw. theoretischen Indifferenz inkorporierte die Politische Ökologie verschiedenste Theorien, Konzepte und Konzeptualisierungen jüngerer wissenschaftlicher Debatten und erneuerte sich dabei stetig. Entscheidende Meilensteine waren etwa die stärkere Berücksichtigung konstruktivistischer und poststruktureller Ansätze (Escobar, 1996; Forsyth, 2003), die Inkorporation von Kenntnissen der Ungleichgewichtsökologie (Botkin, 1990; Moore et al., 1996; Peet und Watts, 1996) und der feministischen Wissenschaftskritik (Haraway, 1988; Rocheleau et al., 1996; Elmhirst, 2011), die Scale-Debatte (Smith, 1984; Marston, 2000; Sayre, 2005; Neumann, 2009) oder die diskursive Betrachtung von Umwelterzählungen (Hajer, 1995; Harré et al., 1999; Adger et al., 2001).

Eine definitorische Abgrenzung dessen, was als Politische Ökologie zu bezeichnen ist, erscheint nahezu unmöglich, kann jedenfalls weder an konkreten Forschungsobjekten oder Problemstellungen noch an bestimmten Maßstabsebenen oder regionalen Verortungen festgemacht werden. Bridge et al. (2015) sehen die einigende Klammer der Politischen Ökologie eher in einer Reihe von gemeinsamen Commitments. Hierzu zählen der theoretische Bezug zur Kritischen Gesellschaftstheorie und ein post-positivistisches Verständnis von Natur und Wissensproduktion, ein methodischer Pluralismus mit Schwerpunkt auf qualitativen empirischen Forschungsmethoden und Berücksichtigung der historischen Dimension, sowie eine normative Verpflichtung mit dem Ziel, soziale

Sybille Bauriedl in diesem Heft), doch eine Fixierung als zentraler Teil von Curricula hat sie gleichwohl (bisher) nicht erfahren. 
Gerechtigkeit und strukturellen politischen Wandel unter Bezugnahme auf die Interessen und Nöte marginalisierter Bevölkerungsgruppen herbeizuführen (Bridge et al., 2015:7-8).

Die Breite der Politischen Ökologie sowie die beachtliche Anzahl an wissenschaftlichen Studien, die sich unter ihrem Label fassen lassen, aber zweifellos auch ihre Fuzziness wurden jüngst durch zwei State-of-the-Art-Publikationen bestätigt: Das Routledge Handbook of Political Ecology (Perreault et al., 2015) wie auch The International Handbook of Political Ecology (Bryant, 2015) vereinen jeweils auf knapp 700 Seiten fast 50 Artikel von etwa fünf Dutzend Autoren. Neben einleitenden (sub)disziplingeschichtlichen Abhandlungen zu Ursprung, Wandlungen und Zukunft der Politischen Ökologie beziehen sich die Beiträge auf Gegenstände, Ansätze, Konzepte, Diskurse und Methoden der Politischen Ökologie. Unbestritten werden in diesen beiden umfang- und inhaltsreichen Bänden zahllose Aspekte jüngerer und aktueller Forschungen im Feld der Politischen Ökologie angesprochen, analysiert und diskutiert - allerdings keineswegs mit einem Exklusivitätsanspruch, denn viele der in diesen beiden Werken behandelten konzeptionellen oder methodischen Problemstellungen wie auch empirischen bzw. praxisrelevanten Forschungsprobleme werden ähnlich auch in anderen Teildisziplinen der Geographie diskutiert; gleichwohl bilden Fragen politischer Einflüsse auf Umwelt und MenschUmwelt-Verhältnisse das allen Beiträgen zu Grunde liegende Hintergrundrauschen. Konzeptionelle, strukturelle und regionale Verknüpfungen lassen sich fast nahezu gleich verteilt mit dem Globalen Norden und dem Globalen Süden in Verbindung bringen. Empirisch regionale Fallbeispiele zeigen jedoch erneut eine sehr ungleiche Verteilung, was erstaunlicherweise sogar in beiden Einleitungskapiteln der Herausgeber explizit formuliert wird: Als zentrale Forschungsregionen der Politischen Ökologie gelten Nord- und Südamerika, Afrika und Europa sowie mit Einschränkungen Asien. Aber Osteuropa, Russland, die Kaukasus-Region oder Zentralasien? Fehlanzeige.

Somit perpetuieren diese jüngsten State-of-the-ArtPublikationen eine bedeutende Leerstelle der Politischen Ökologie, indem sie ein anderes ,,in-between“ vernachlässigen, nämlich die sozialistische und postsozialistische Welt. Die Herausgeber und Autoren beider Bände wie auch die meisten Politischen Ökologen schenken der (post)sozialistischen Sphäre wenig bis keine Aufmerksamkeit. Fast zynisch wiesen bereits Watts (1990) und Engel-Di Mauro (2009) darauf hin, dass die meisten Politischen Ökologen gar nicht fähig seien, die Unterschiede zwischen kapitalistischen und sozialistischen Gesellschaften zu erkennen oder gar die damit verbundene notwendige ,transideologische“ Herausforderung zu bewältigen; sozialistische Gesellschaften würden nur als von der kapitalistischen Weltwirtschaft isolierte Relikte gesehen. Und gerade auch die spannende Frage, inwiefern ehemals sozialistische Gesellschaften mit dem materiellen und immateriellen Erbe des Sozialismus umgehen, wird nur in wenigen Studien aufgegriffen.

Doch woher rührt diese mangelnde Berücksichtigung, gar Ignoranz gegenüber der (post)sozialistischen Sphäre? Zeichnen sich hierfür wissenschaftstheoretische, forschungspolitische oder einfach nur praktische Gründe verantwortlich? Um Missverständnissen und Widersprüchen vorzubeugen: Keineswegs bleiben Umweltdegradationen, Mensch-UmweltBeziehungen oder Machtasymmetrien bei der Auseinandersetzung mit Natur und natürlichen Ressourcen im postsozialistischen Raum wissenschaftlich unbeachtet. Vielmehr existiert eine zahlenmäßig beachtliche und aktive internationale Scientific Community, die sich neben vielen anderen Fragestellungen auch mit den genannten Forschungsproblemen im Bereich Mensch und Umwelt in der ehemaligen Zweiten Welt auseinandersetzt. Hierunter fallen auch einige explizit politisch-ökologische Studien in postsozialistischen Räumen, wie etwa Muldavin $(1997,2008)$ zum ländlichen Raum Chinas, Harper (2006) zu Umweltbewegungen in Ungarn, Hartwig (2007) zur Ressourcennutzung in der Mongolei, Staddon (2009) zu Mensch-Forst-Beziehungen in Bulgarien, Schmidt (2013) zum Wald- und Ressourcenmanagement in Kirgistan oder Dörre (2014) zu Weidelandfragen in Kirgis$\tan$.

Doch eine konzeptionelle Auseinandersetzung mit den postsozialistischen Partikularitäten findet in der Politischen Ökologie nicht statt. Vor dem Hintergrund ihres theoretischen Bezugs auf Marxismus und Neomarxismus steht insbesondere die Kritik an Kapitalismus und westlicher Hegemonie im Fokus politisch-ökologischer Studien, während die Interdependenzen von Ressourcenmanagement, Marginalisierung und Umweltwandel in den sozialistischen Staaten und den postsozialistischen Folgerepubliken weitgehend unberücksichtigt bleiben. Die führenden, den Diskurs der Politischen Ökologie bestimmenden Proponenten scheinen diesen in historischer wie auch politischer Hinsicht durchaus bemerkenswerten und bedeutsamen Raum als für die Weiterentwicklung der Politischen Ökologie irrelevant oder für ihre wissenschaftliche Auseinandersetzung als zu problemgeladen zu betrachten - zumindest deutet das Fehlen entsprechender wissenschaftlicher Publikationen darauf hin.

\section{Gründe für eine Beschäftigung der Politischen Ökologie mit dem Postsozialismus}

Die Debatten und konzeptionellen Weiterentwicklungen der Politischen Ökologie wurden und werden von Wissenschaftlern des anglo-amerikanischen Raumes dominiert. Deren Fokus richtet sich neben ihrem eigenen Lebensumfeld verstärkt auf Nord- und Südamerika sowie auf ehemalige Kolonien in Afrika und Asien. Eurasien im Sinne von Mittel- und Osteuropa, der Kaukasus-Region, Nord-, Zentral- und Ostasien wird dagegen selten bearbeitet. 
Obgleich viele Vertreter der Politischen Ökologie marxistische bzw. neomarxistische Argumentationen bemühen (Watts, 1983; Blaikie, 1985; Peet und Watts, 1996; Forsyth, 2003; Castree, 2015), werden Umwelt und Gesellschaften des ehemals real existierenden Sozialismus selten als Forschungsfeld in Betracht gezogen. Hier drängt sich die fast zynische Frage auf, ob dies damit zusammenhängen könnte, dass sich die theoretischen Vorzüge und Logiken des Marxismus mit ihrer - zweifellos berechtigten - Kritik am dominierenden Kapitalismus nicht gut mit den Realismen eines gescheiterten real existierenden Sozialismus vertragen. Möglicherweise könnten die negativen und stellenweise katastrophalen Auswüchse - etwa die Gewaltexzesse des Stalinismus - und gescheiterten Ideale des real existierenden Sozialismus die eigene, positiv konnotierte marxistische Argumentation schwächen. Tatsächlich erscheint diese Vermutung zweifelhaft, da gerade das kritische Hinterfragen von vermeintlichen Essentialismen in der Politischen Ökologie einen hohen Stellenwert einnimmt. Vielmehr sind die Gründe eher in forschungspolitischer und praktischer Hinsicht zu suchen: Traditionelle koloniale Verbindungen, aktive soziale und ökologische Bewegungen in Lateinamerika, Sprachkompetenzen bzw. -barrieren, aber auch die Zielrichtung von Forschungsgeldern mögen stichhaltigere Begründungen liefern.

Warum aber lohnt sich überhaupt eine politischökologische Beschäftigung mit der postsozialistischen Sphäre? Die Tatsache, dass eine bestimmte Region mit einem spezifischen Forschungsansatz nicht beforscht würde und deshalb dringender Forschungsbedarf bestehe, ist sicherlich kein gutes und schon gar kein zwingendes Argument für eine zu leistende wissenschaftliche Auseinandersetzung. Schließlich gibt es beispielsweise keine oder kaum politisch-ökologische Studien zu Mauretanien, Tonga oder Brandenburg. Aber, die hier beklagte Ignoranz betrifft immerhin einen Raum von 30 Staaten und - unter Einschluss Vietnams und der Volksrepublik China - fast zwei Milliarden Menschen, deren historisches Erbe und damit auch ihre Gegenwart sich signifikant von jenen anderer Weltregionen unterscheidet und partikuläre Gemeinsamkeiten - den (Post)Sozialismus - aufweist. Eine Politische Ökologie, die vorurteilsfrei Machtkonstellationen von Mensch-Umwelt-Interaktionen kritisch hinterfragt, die mit empirischen Methoden Strukturen, Prozesse und Handlungen auf unterschiedlichsten Maßstabsebenen und deren Wechselwirkungen analysiert, und die schließlich auch mit einem normativen Anspruch aktiv gestalten will, kann sich die Vernachlässigung eines solch relevanten Raumes nicht leisten.

\subsection{Postsozialismus als Kategorie}

Neben dem genannten Argument der großen Zahl an betroffenen Staaten und Menschen bzw. der weltregionalen Dimension ist allerdings auch zu prüfen, ob und warum Postsozialismus als Kategorie für politisch-ökologische Betrach- tungen von Interesse ist. Hierfür ist zunächst zu klären, was unter Postsozialismus zu verstehen ist und warum es heute, 25 Jahre nach dem Ende des sozialistischen Experiments noch eine relevante analytische Kategorie ist. ${ }^{2}$

Unter Postsozialismus ist zunächst jene Epoche zu verstehen, die sich an den (real existierenden) Sozialismus anschließt, worauf das Präfix ,post“" verweist. Ein Verständnis im Sinne eines temporären „danach“ greift jedoch zu kurz, weil es impliziert, es würde sich um eine Ablösung der vorhergegangenen Epoche handeln. Vielmehr ist jedoch in Analogie zum Ansatz des Postkolonialismus (Nash, 2002; Loomba, 2005) von einer Persistenz sozialistischer Diskurse wie auch politischer, ökonomischer und gesellschaftlicher Strukturen, sprich von einer fortdauernden Durchdringung und Prägung des Jetzt durch das Gewesene auszugehen. Ähnlich wie der Postkolonialismus ohne den Kolonialismus undenkbar ist und explizit die Legacies des Kolonialismus aufzeigt, ist auch der Postsozialismus ohne den Sozialismus nicht zu verstehen.

Materielle und institutionelle Infrastrukturen, Naturräume und Gesellschaften wurden im Zuge der sozialistischen Modernisierung im 20. Jahrhundert maßgeblich gestaltet, geprägt und transformiert. Die heutigen postsozialistischen Gesellschaften lassen nicht nur Spuren dieser Prozesse erkennen, sondern bauen auf diesen Strukturen auf. Ungeachtet radikaler Wechsel der Herrschaftsverhältnisse, juristischer Reformen, technologischer Innovationen oder Einflüssen der Globalisierung sind die Erfahrungen von Jahrzehnten sozialistischen Zusammenlebens fest im kulturellen Gedächtnis verankert, erweisen sich gesellschaftliche Wandlungen als träge und bestehen technische und institutionelle Infrastrukturen in unterschiedlichem Grade fort bzw. konnten oder können nur schrittweise modifiziert oder ersetzt werden. Ganz plastisch ausgedrückt, die Materialitäten des Sozialismus sind in Osteuropa oder Zentralasien auch heute noch sicht- und spürbar, etwa in Form von Plattenbausiedlungen, Aufmarschalleen oder gewaltigen Industriekomplexen, aber auch in Form von institutionellen Strukturen, individuellen Erinnerungen an staatssozialistische Auszeichnungen, wie sich dies etwa durch die Zurschaustellung von Medaillen und Plaketten bei offiziellen Gedenkfeiern manifestiert, oder die Orientierung nach Moskau, was durch den regelmäßigen Konsum russischer Nachrichten in den ehemaligen Sowjetrepubliken oder die gegenwärtigen Migrationsbewegungen in Richtung Russland deutlich wird.

Für Hann (2002:7) bleibt die Kategorie ,postsozialistisch“ so lange relevant, wie die „Ideale, Ideologien und Praktiken des Sozialismus für das Verständnis der gegenwärtigen Lage den betroffenen Menschen als Bezugspunkt dienen" und für ihr heutiges Handeln strukturierend wirken. Auch Humphrey (2002:26) betont die fortbestehende Rele-

\footnotetext{
${ }^{2}$ Das Konzept der Transformation bleibt hier bewusst unberücksichtigt aufgrund seiner Unschärfe und der inhärenten teleologischen Konnotationen (Schmidt, 2013).
} 
vanz der Kategorie „postsozialistisch“, weil der real existierende Sozialismus eine ,zutiefst durchdringende Erscheinung“" war, die nicht ,plötzlich und total“ verschwindet und durch eine völlig andere Lebensweise ersetzt werden könne. Demnach ist Postsozialismus nicht nur ein wissenschaftliches Konstrukt, sondern erlebte Realität auf Grundlage historischer Entwicklungen. Entsprechend müssen bei einer politisch-ökologischen Analyse gegenwärtiger MenschUmwelt-Verhältnisse die real-sozialistische Geschichte und deren Materialitäten angemessen Berücksichtigung finden.

\subsection{Forschungsfelder einer Politischen Ökologie des Postsozialismus}

Umweltprobleme im (post)sozialistischen Raum sind prävalent. Die Reaktorkatastrophe von Tschernobyl mag als die prominenteste Umweltkatastrophe im „Ostblock“ stehen es ist zweifellos nicht die einzige. Hier sei nur auf den ausgetrockneten Aralsee, die radioaktive Verseuchung von Nowaja Semlja und Semipalatinsk oder ungezählte Leckagen bei der Öl- und Gasförderung in Nordrussland verwiesen. Die Auseinandersetzung mit solcherart Umweltproblemen, mit anthropogenen Ursachen und Auswirkungen oder die Behandlung politischer Aushandlungsprozesse samt deren Folgen für marginalisierte Gruppen sind Kernbestandteil politisch-ökologischer Studien. Gleichwohl beklagen Peet und Watts (1996:4) die mangelnde Berücksichtigung dieses „, terrifying environmental record“ der sozialistischen Regionen durch die Politische Ökologie.

Wie gezeigt, fallen heute noch wesentlich weiter gefasste wissenschaftliche Problem- und Themenstellungen in das Feld der Politischen Ökologie, von denen sich Entsprechungen in beliebig großer Zahl auch im postsozialistischen Raum finden. Beispielsweise wurde Naturschutz keineswegs alleine im Westen verhandelt. Vielmehr standen der Schutz unberührter Naturlandschaften und die Einrichtung von Schutzgebieten auch in der Sowjetunion auf der Agenda (vgl. Meessen, 1992). Heute wirkt dieses Erbe nach, nicht nur im Hinblick auf die gegenwärtige Nutzung dieser Areale, sondern etwa auch im Bezug auf die Wahrnehmung und Konzeptionen von Natur (Dörre und Schmidt, 2008). Auch der in den letzten Jahren stark an Popularität gewonnenen Urban Political Ecology bieten sich zahlreiche Anknüpfungspunkte für Studien in den durch Plattenbauten, Kommunalwohnungen (Kommunalka) und sozialistische Stadtplanung geprägten, heute durch rege Bautätigkeiten, illegale Zuwanderung, Errichtung abgeschlossener Wohnanlagen und wachsende Ungleichheit gekennzeichneten städtischen Räumen Osteuropas. Aushandlungsprozesse zwischen verschiedenen Akteuren beim Ressourcenmanagement, Machtasymmetrien zwischen Mitgliedern der ehemaligen sozialistischen Nomenklatura und Aufsteigern oder Verlierern gegenwärtiger Entwicklungen, Entscheidungsprozesse in Regional- oder Stadtplanung, aber auch die Beschäftigung mit der Persistenz oder Transformation sozialistischer
Diskurse sind weitere fruchtbare Forschungsfelder für eine Politische Ökologie des Postsozialismus.

Die große Bandbreite an möglichen Fragestellungen und Forschungsfeldern ist unbestritten, gleichwohl ist zu klären, welchen Erkenntniswert eine explizit politisch-ökologische Untersuchung postsozialistischer Gesellschaften und Umwelten bietet und inwiefern die Strukturen und Prozesse in postsozialistischen Räumen sich signifikant von jenen in anderen Weltregionen unterscheiden.

\section{Partikularitäten postsozialistischer Gesellschaft-Umwelt-Verhältnisse}

In dem durch ideologische Konfrontationen geprägten „,kurzen 20. Jahrhundert" (Hobsbawm, 1995) unterschieden sich die politischen, wirtschaftlichen und gesellschaftlichen Systeme des Westens und Ostens signifikant. Auch das MenschUmwelt-Verhältnis differierte und prägte entsprechend $\mathrm{Na}-$ turräume, Wirtschaft und Gesellschaft und damit auch die Geographie. Studien in postsozialistischen Räumen kommen deshalb nicht umhin, die jüngere Geschichte zu berücksichtigen. Denn ohne den Blick auf die Socialist Legacies bleiben gegenwärtige Strukturen, Prozesse und Probleme unverständlich. Die heutigen Mensch-Umwelt-Verhältnisse Eurasiens sind wesentlich durch die Vergangenheit geprägt, bauen auf ihr auf und zeigen spezifische (postsozialistische) Merkmale, wie - ohne Anspruch auf Vollständigkeit und Allgemeingültigkeit - im Folgenden erläutert wird.

\subsection{Vom allmächtigen zum schwachen Staat}

Im Gegensatz zu den von Neoliberalen und Neomarxisten beklagten staatlichen Überregulierungen und Interventionen ist in den postsozialistischen Staaten ein gegenläufiger Prozess zu beobachten, nämlich die Schwächung eines zuvor dominanten, regulierenden, kontrollierenden, aber auch sorgenden Staates. Die sozialistischen Gesellschaften und Ökonomien waren durch einen hohen Institutionalisierungsgrad gekennzeichnet. Parteiorgane und Administrationen durchdrangen weite Bereiche des wirtschaftlichen und gesellschaftlichen Lebens, während private Aktivitäten erheblich eingeschränkt, kontrolliert und reglementiert waren. Eigentumsrechte, Kontrolle und Bewirtschaftung von Land- und $\mathrm{Na}-$ turressourcen lagen fast ausnahmslos in staatlicher Hand. Im ländlichen Raum beispielsweise dominierten auf lokaler Ebene Staats- (Sowchoz) und Kollektivbetriebe (Kolchoz) das politische, wirtschaftliche und auch gesellschaftliche Leben (Lindner, 2008). Durch das staatliche Bildungssystem und intensive Propagandamaßnahmen beeinflusste der Staat auch die Deutung von Natur und Umwelt, die es zu bezwingen und zu bändigen galt (Bolotova, 2008; Féaux de la Croix, 2014).

Mit dem Ende des sozialistischen Experiments 1989/91 setzte eine Erosion staatlicher Institutionen ein und damit eine Schwächung der Zentralstaaten, ganz besonders aus- 
geprägt in den jungen, aus der Erbmasse der Sowjetunion hervorgegangen Republiken des Kaukasus und Zentralasiens. Fehlende Finanzmittel, die Auflösung des Rats für gegenseitige Wirtschaftshilfe (RGW) und damit der Wegfall wirtschaftlicher Unterstützung und Austauschprozesse sowie die mühsame Umgestaltung politischer und administrativer Strukturen führten zu einem Rückgang des Staatseinflusses, zur Unfähigkeit territorialer und gesellschaftlicher Kontrolle sowie zu einer deutlichen Kürzung von Staatsausgaben für Bildung, Gesundheit und andere Sozialleistungen. In weiten Teilen der Bevölkerung resultierten diese Entwicklungen in Armut, zunehmender Verwundbarkeit sowie einem Gefühl von Unsicherheit, Destabilisierung und Marginalisierung (Steimann, 2011; Dörre, 2014). Dies betraf in den frühen 1990er Jahren nahezu ausnahmslos alle postsozialistischen Staaten, die sich gleichwohl seither sehr unterschiedlich entwickelten.

In seiner Bedeutung nicht zu vernachlässigen ist zudem der plötzliche Verlust einer das Alltagsleben durchdringenden Ideologie, die quasi über Nacht als falsch und gescheitert betrachtet wurde und nur schwer durch neue Ideologien ersetzt werden konnte. Seither bemühen sich die Herrschenden der jungen Staaten darum, im Prozess des Nation Building neue gesellschaftliche Leitlinien zu entwickeln - mit nicht unerheblichen neuen Problematiken angesichts zahlreicher Bevölkerungsminderheiten, die nicht der in diesem Diskurs positiv hervorgehobenen Titularethnie angehören (Schmidt, 2007). Diese Staatsschwäche, die Mühen des Nation Building sowie die Unsicherheiten in materieller wie auch ideologischer Hinsicht sind bei Studien der Gesellschaft-UmweltVerhältnisse im postsozialistischen Raum näher zu berücksichtigen.

\subsection{Sozialistische Modernisierung}

Modernisierung erfolgte in der Sowjetunion und ab 1945 auch in Osteuropa nach sozialistischen Leitlinien und nicht nach westlich-kapitalistischem Modell. Mit seiner Parole „Kommunismus ist Sowjetmacht plus Elektrifizierung“ wies Lenin bereits im November 1920 auf das Modernisierungsprojekt der im Entstehen begriffenen Sowjetunion hin. Es besteht kein Zweifel, dass im sozialistischen Osteuropa und in der Sowjetunion im Laufe des 20. Jahrhunderts gewaltige Modernisierungsanstrengungen unternommen wurden. Diese umfassten die Förderung formaler Bildung, den Aufund Ausbau materieller und institutioneller Infrastrukturen, Industrialisierung, Emanzipation sowie die Zurückdrängung von Religion und Tradition. Obgleich viele Parallelen zur westlich-kapitalistischen Modernisierung bestehen und als „evolutionäre Universalien“ (Parsons, 1972) gelten können wie etwa bürokratische Organisation und Schaffung eines Recht- und Geldsystems, wies die sozialistische Modernisierung durchaus abweichende Ziele auf. Demnach lag die Präferenz nicht auf individueller Selbstentfaltung oder Etablierung bürgerlicher Demokratie, sondern auf dem Einbringen individueller Fähigkeiten und Bemühungen in ein Kollektiv mit all ihren Folgen für das Mensch-Umwelt-Verhältnis. Vor diesem Hintergrund sollten die Wechselwirkungen zwischen sozialistischer Modernisierung und real existierendem Sozialismus auf der einen Seite und Umweltwandel, Ressourcennutzung oder Naturschutz auf der anderen Seite betrachtet werden.

\subsection{Modernisierte heterogene Gesellschaften}

In ethnographischen Studien zum Mensch-UmweltVerhältnis lokaler Gemeinschaften im Globalen Süden, insbesondere aus den Anfangsjahren der Politischen Ökologie, findet oftmals eine Beschwörung idealisierter, sozio-kulturell homogener „lokaler Gemeinschaften“ statt. Die Suche nach dem Ursprünglichen und Unverfälschten führte nicht selten zu einem „romantischen Essentialismus“ (Forsyth, 2003). Eine solche Suche bliebe in den postsozialistischen Gesellschaften und Umwelten mit größter Wahrscheinlichkeit erfolglos. Denn wie eben gezeigt vollzog sich der jüngste kapitalistische Umbau des Wirtschaftssystems nicht in prämodernen Gesellschaften, sondern in sozialistisch modernen Staaten. Obgleich das Ideal des Homo Sovieticus und der proletarische Internationalismus auf eine Angleichung von Verwirklichungschancen und eine Nivellierung regionaler und insbesondere nationaler Unterschiede abzielte, sprich eine Homogenisierung von Gesellschaften, Lebensstilen und Lebensweisen anstrebte, fand sie in heterogenen, oftmals multiethnischen und durchaus auch konfliktgeladenen Gesellschaften statt. Unterschiede in Sprache, Kultur und Tradition konnten nirgends komplett nivelliert werden und wurden stellenweise im Zuge nationaler Selbstbestimmungsansprüche in der zweiten Hälfte des 20. Jahrhunderts partiell hervorgehoben. Mit der Gründung neuer Nationalstaaten erfolgte als Prozess des Nation Building eine Bestärkung nationaler einigender Merkmale, aber auch eine Betonung der Differenzen gegenüber anderen (Schmidt, 2007; Marat, 2008).

Die Arbeitsteilung und Spezialisierung innerhalb größerer Kollektive führte zu einem oftmals verengten Blick auf das Ganze. Externe Interventionen, die Übertragung und Beschränkung von Zuständigkeiten in einem hierarchisch aufgebauten System bewirkten einen Mangel an Verantwortungsbewusstsein und Eigeninitiative sowie eine Entfremdung von der umgebenden Natur bzw. den lokalen Naturressourcen. Auch die gewaltigen und zumeist gewaltsamen An- und Umsiedlungsprogramme trugen dazu bei, dass traditionelles lokales Wissen verloren ging oder sich ein von individuellen Interessen geprägtes enges Verhältnis zwischen Gesellschaften und lokaler Umwelt kaum bilden konnte (Schmidt, 2013). Somit müssen politisch-ökologische Studien in postsozialistischen Kontexten Idealisierungen, aber auch Vereinheitlichungen und damit Vereinfachungen vermeiden. 


\subsection{Umweltnarrative und hegemoniale Naturschutzdiskurse}

Ressourcenbedürfnisse und Vorstellungen über den ,richtigen" Umgang mit Umwelt und Natur sind zeit- und kontextabhängig. Entsprechend weist auch der Sozialismus spezifische Vorstellungen zum Verhältnis von Mensch und Umwelt auf. Die Sowjetunion sah sich selbst als Vorreiterin des Naturschutzes und sprach dem Kapitalismus aufgrund seines inhärenten Motivs der Eigennutzorientierung jegliche Fähigkeit zum Naturschutz ab (Dörre und Schmidt, 2008). In der sozialistischen Ära wurden in Osteuropa und der Sowjetunion zahlreiche Schutzgebiete eingerichtet (Meessen, 1992). Neben den Zapovedniki, in denen ähnlich dem westlichen Nationalparkkonzept jegliche menschliche Nutzung untersagt war, war in den Zakazniki räumlich und zeitlich beschränkt eine ,sozialistische Naturnutzung “ möglich. Grundsätzlich sollte im Sozialismus der Naturschutz dem Erfolg des sozialistischen Projektes dienen.

Die Entwicklungen von Umweltmythen und hegemonialen Diskursen zum Naturschutz bilden allzu oft die Basis für scheinbar objektive, apolitische Konzepte. Dies trifft auch auf den (post)sozialistischen Raum zu, wo zunächst andere Vorstellungen als in westlich-kapitalistischen Gesellschaften dominierten (Bolotova, 2008), die aber heute vielerorts von den derzeit global wirkmächtigen Schutzdiskursen abgelöst werden und die Debatten in den postsozialistischen Staaten beeinflussen. Die Nutzung der entsprechenden Termini wie Biodiversität oder Nachhaltigkeit etc. selbst auf lokaler Ebene, wie etwa im Forstmanagement Südkirgistans beobachtet (Dörre und Schmidt, 2008), zeugen davon.

Externe Einflussnahmen äußern sich aber nicht nur in hegemonialen Diskursen, sondern auch in handfester Landpolitik: Zum einen finden verschiedenste Prozesse der Kommodifizierung von Natur und Umweltelementen statt, seien es die Artenvielfalt, Landschaftsschönheit oder Heilpflanzen. Zum anderen nimmt die Aktivität des Landmarktes an Fahrt auf, externe Landakquisitionen (Land Grabbing) sind insbesondere in Russland und Kasachstan prävalent (Visser und Spoor, 2011). Aufgrund der unterschiedlichen Geschichte von staatlicher Beeinflussung und Dominanz im Landmanagement sind die Vorzeichen andere als beispielsweise in Subsahara-Afrika. Während Land Grabbing in Sub-SaharaAfrika oftmals mit staatlichen Modernisierungsprogrammen und einer faktischen Enteignung pastoraler Gruppen einhergeht (Schmidt und Pearson, 2016), bieten sich in Russland und Kasachstan besonders Räume an, die von Bevölkerungsrückgang und Abwanderung betroffen und in den vergangenen Jahren aus der Produktion gefallen sind (Visser und Spoor, 2011). Diese andersartigen Vorzeichen, die sich entsprechend auch auf die damit einhergehenden Kommodifizierungsprozesse auswirken, bieten Anknüpfungspunkte für erkenntnisreiche politisch-ökologische Untersuchungen.

Unter Bezugnahme auf die Debatten innerhalb der Politischen Ökologie und der hier dargestellten Partikularitäten in postsozialistischen Räumen sollen im folgenden Abschnitt Prinzipien einer Politischen Ökologie des Postsozialismus skizziert werden. Allerdings ist vor vereinheitlichenden Aussagen zu warnen. Denn der hier behandelte Raum weist zwar gemeinsame Elemente wie den (Post)Sozialismus auf, ist jedoch sowohl in historischer als auch gesellschaftlicher Hinsicht äußerst heterogen.

\section{Prinzipien einer Politischen Ökologie des Postsozialismus}

Eine Politische Ökologie des Postsozialismus kann und sollte auf den bisher geführten zentralen Ansätzen und Debatten der Politischen Ökologie aufbauen. Allerdings erfordert sie Ergänzungen und Modifikationen sowie stellenweise Neujustierungen von Prämissen. Prinzipiell sollte sie den Fokus auf Strukturen und Prozesse in Politik, Ökonomie und Gesellschaft ebenso legen wie auf poststrukturelle Aspekte wie die Konstruktion von Umweltnarrativen, Naturschutzkonzepte oder Diskurse zum Umweltwandel.

\subsection{Sozialistische und postsozialistische Modernisierung im Diskurs}

In Analogie zur Post-Development-Debatte (Escobar, 1995) mit ihrer fundamentalen Kritik am Entwicklungsmodell gilt es, den Fortschrittsglauben der sozialistischen Modernisierung kritisch zu überprüfen und zu dekonstruieren. Die dem Marxismus inhärente Vorstellung einer linearen Entwicklung in historischen Stufen sowie der auf Aufklärung und Rationalismus aufbauende Glaube an Szientismus waren prägend für den sozialistischen Modernisierungsdiskurs. Das Zurückdrängen von Traditionen und Religion, der Aufbau von Gesundheits- und Bildungssystemen, Emanzipierung, Industrialisierung und Ausbau von materiellen und institutionellen Infrastrukturen waren stets positiv belegt (Nove, 1980). Dennoch gilt diese Modernisierung aus heutiger, westlicher Sicht als gescheitert. Stattdessen sollten dem $\mathrm{Wa}$ shington Consensus zufolge Liberalisierung, Privatisierung und Demokratisierung zu einer Entfesselung individueller Kräfte und Wohlstand führen (World Bank, 1996). Demnach stellen Modernisierung und Neoliberalisierung nach westlichen Idealen nicht Ursache von Verarmung und Umweltdegradation dar, sondern deren Lösung.

Eine Politische Ökologie des Postsozialismus muss sowohl die Erfolge und Gründe des Scheiterns sozialistischer Modernisierung als auch die Probleme westlichkapitalistischer Modernisierung analysieren und deren Verantwortung etwa an gegenwärtigen Umweltproblemen und den damit zusammenhängenden gesellschaftlichen Marginalisierungen herausarbeiten. Entsprechend muss die positive Konnotation von Modernisierung als lineare, aufwärts gerichtete Entwicklungsvorstellung kritisch überprüft werden, die sich sowohl in offiziellen Strategiepapieren als auch in den meisten Köpfen der durch diese Leitbilder beeinfluss- 
ten Bevölkerungen findet. Denn ebenso wenig wie das sozialistische Fortschrittskonzept hinterfragt wurde, fand in den ersten Jahren nach der Wende eine kritische Reflexion der Vorstellungen von nachholender Entwicklung oder anzustrebender Konvergenz mit dem Westen statt. Erst in jüngster Zeit setzte eine kritische Auseinandersetzung oder gar Ablehnung westlicher Ideale ein, wie dies etwa die nationalkonservativen Regierungen in Russland, Ungarn oder Polen prominent betreiben. Tatsächlich ist jede Neuausrichtung eines Entwicklungspfades mit der Marginalisierung von Gruppen oder Handlungsweisen verbunden, die nicht in das Neue passen. Demnach muss eine Politische Ökologie des Postsozialismus die mit dem jeweiligen Modernisierungsparadigma verknüpften ökologischen, ökonomischen und gesellschaftlichen Kosten berücksichtigen.

Überwiegend wird in politisch-ökologischen Studien der Kapitalismus als wesentliche Ursache für Umweltzerstörung und rücksichtslose Ausbeutung von Naturressourcen gesehen; ein Gedanke, der sich auf die Kritische Theorie der Frankfurter Schule zurückverfolgen lässt (Forsyth, 2003). Dem Kapitalismus zufolge ist Natur Kapital, das geerntet und ausgebeutet werden kann. Natur wird als eine Ansammlung von Ressourcen konstruiert, die für das globale Wirtschaftssystem unverzichtbar sind (Escobar, 1996). Demgegenüber wird nach der Dialektik von Marx die als ,,chaotisch, sinnfrei (...) feindlich, fremd und wild" (Bolotova, 2008:58) charakterisierte Natur durch die Aktivitäten des Menschen in Wert gesetzt. Die ökologischen Folgen dieser Maxime waren in den Staaten des real existierenden Sozialismus jedoch keineswegs weniger verheerend als in kapitalistischen Regionen. Allerdings sind diese Umweltprobleme nicht ursächlich auf kapitalistische Profitmaximierung zurückzuführen, sondern wohl eher eine Folge der ,economics of shortage" (Kornai, 1980) und der (Ir)Rationalitäten zentralistischer und ideologiegesteuerter Staatsplanung mit ihrer Präferenz von Schwerindustrie und industriellem Gigantismus (Peet und Watts, 1996).

Eine Konzeptionalisierung der Zusammenhänge und Wechselwirkungen von Sozialismus und Umweltwandel fand in der Politischen Ökologie bisher nicht statt. Zwar wies bereits Blaikie (1985) auf die ideologischen Differenzen zwischen „,zentralen Planwirtschaften“ und „fortschrittlichen kapitalistischen Staaten" hin, versäumte es jedoch, Gemeinsamkeiten oder Unterschiede im Naturschutz, Umwelt- oder Ressourcenmanagement zu analysieren. Aufgabe einer Politischen Ökologie des Postsozialismus sollte es demnach sein, Umweltdegradation nicht nur als Folge kapitalistischer Ausbeutung zu sehen, sondern auch als Resultat des real existierenden Sozialismus zu beleuchten.

\subsection{Dekonstruktion sozialistischer und postsozialistischer (Umwelt) Narrative}

Umwelt, Natur oder Ressourcen sind keine ontologischen Gegebenheiten, sondern erst durch anthropogene Zuschrei- bungen, Klassifikationen und Grenzziehungen geschaffen (Bridge, 2009). Dabei legen die jeweiligen Gesellschaften und Ökonomien fest, welche naturräumliche Entität oder Leistung als Ressource bzw. Dienstleistung zu nutzen und welche Elemente oder Räume als „Natur“ zu schützen sind. Ressourcenbedürfnisse wandeln sich mit den politischen Herrschaftsverhältnissen oder den ökonomischen Systemen und Bedürfnissen. In vorsozialistischer Zeit verfolgten die herrschenden Feudalsysteme andere Ziele der Ressourcenausbeutung als etwa die Planwirtschaft des Staatssozialismus. Analog sind Leitlinien des ,richtigen“ Umgangs mit oder Schutzes der Natur ebenfalls zeit- und kontextabhängig und wurden mit Bedeutungszunahme wissenschaftlicher Erkenntnisse durch gezielte Interpretationen und Daten untermauert, was zur Ausbildung nicht weiter hinterfragter Umweltmythen führte. In gleicher Weise etablierten sich vermeintlich objektive und apolitische Konzepte wie "moderne“ Landwirtschaft, ,optimierte“ Zucht oder ,effiziente“ Produktion zu hegemonialen Diskursen.

Eine normative Politische Ökologie des Postsozialismus sollte solche Konstruktionen aufdecken, deren Einfluss auf Umwelt und Gesellschaft herausstellen und am Wahrheitsgehalt von wissenschaftlich oder staatlich definierten Kategorien zweifeln, die oftmals willkürlich gewählt waren, um alternative Interpretationen zurückzudrängen und politische Maßnahmen zu legitimieren.

Im gegenwärtigen dominierenden Naturschutzdiskurs fungiert der Mensch als Akteur, der das vermeintliche ökologische Gleichgewicht stört und Artenrückgang und Naturdegradation herbeiführt (Zimmerer und Bassett, 2003). Die Bewahrung von Natur, der Erhalt von Biodiversität oder der Schutz des Klimas unter dem Mantra der Nachhaltigkeit sind demnach von globalem Interesse, was nicht selten mit an räumliche Gegebenheiten angepassten und über lange Zeiträume produktiven und stabilen Systemen der Landund Naturnutzung kollidiert und zu deren Destabilisierung oder gar Eliminierung führt, da sie von mächtigeren Akteuren im Wettstreit um die Kontrolle von Ressourcen als den genannten Zielen nicht dienlich charakterisiert werden (Robbins, 2012). Ein solcherart verstandener Naturschutz ist folglich auch eine Form hegemonialer Machtausübung.

Damit steht eine Politische Ökologie des Postsozialismus vor der Herausforderung, die heute in den postsozialistischen Staaten nur noch schwach ausgeprägten traditionellen Formen des Umweltmanagements zu identifizieren und gegebenenfalls zu fördern oder in dominantere institutionelle Systeme zu integrieren. Allerdings ging durch die sozialistische Modernisierung, durch Zurückdrängen von Traditionen, durch Arbeitsteilung und Spezialisierung ein großer Teil autochthonen Wissens verloren und kann auch nicht im $\mathrm{Zu}-$ ge falsch verstandener Romantisierung präsozialistischer Bedingungen reanimiert werden. 


\subsection{Nüchternheit statt Romantisierung}

Jüngere Entwicklungsprogramme und Formate wie Соттиnity Based Natural Resource Management (CBNRM) propagieren ein gemeinschaftlich orientiertes Naturressourcenmanagement sowie die Berücksichtigung lokalen Wissens (Berkes, 1999; Neumann, 2005). Das „Lokale“, „Indigene“ oder die "Gemeinschaft“" werden als Teil einer nachhaltigen, sanften und lokal angepassten Lösung hervorgehoben, basieren vielfach jedoch auf einer naiven Idealisierung und Romantisierung. Dies geht einher mit Zweifeln und Kritik an staatlichen Institutionen, wie sie auch von Vertretern des Neoliberalismus geäußert werden. Die Beschwörung einer idealisierten, durch gesellschaftliche und kulturelle Homogenität sowie einen großen Reichtum an lokalem Wissen geprägten „lokalen Gemeinschaft“ wird von Vertretern einer poststrukturalistischen Politischen Ökologie kritisiert (Forsyth, 2003; Neumann, 2005).

Im postsozialistischen Raum entsprechen die lokalen Gemeinschaften keineswegs diesem Ideal. Heutige Dorfgesellschaften sind viel eher heterogen und konfliktgeladen und schon gar nicht unbeeinflusst von historischen Entwicklungen und externen Interventionen. Modernisierungsmaßnahmen, berufliche Spezialisierung auf eng umrissene Tätigkeitsbereiche, hierarchisch aufgebaute Entscheidungs- und Verantwortungsketten sowie massive An- und Umsiedlungsmaßnahmen führten zu einer Entfremdung von der lokalen Umwelt und resultierten in heute eher limitiertem Umweltwissen. Naturressourcen wurden im 20. Jahrhundert nicht nach autochthonen Formen gemeinschaftsorientiert bewirtschaftet, sondern in staatlich implementierten Kollektiven mit zentralistisch determinierten Regularien.

\subsection{Berücksichtigung institutioneller Rekonfigurationen}

Im Fokus politisch-ökologischer Studien stehen oftmals Analysen von Besitz- und Verfügungsrechten an Naturressourcen (Robbins, 2012; Blomley, 2015), die Art, wie sie zwischen verschiedenen gesellschaftlichen Gruppen verhandelt werden, wer davon profitiert oder benachteiligt wird. Eine solche Analyse dient dazu, den Zusammenhang von Property Rights mit Umweltdegradierung und Marginalisierung herauszustellen. Dabei können sich Besitz- und Nutzungsrechte an Land oder Naturressourcen überschneiden und widersprechen, zudem sind sie vielfach umstritten und umkämpft.

Zugang zu Ressourcen ist ein bedeutsamer Faktor für Entwicklungsprozesse. Nach marktwirtschaftlicher Logik internalisiert Privatisierung Kosten und Gewinne und führt zu einer rationalen Ressourcennutzung (McCay und Acheson, 1987). Änderung von Besitzregimen führen jedoch oftmals zu einer „tragedy of enclosure“ (Bryant und Bailey, 1997) und zum Ausschluss bestimmter Nutzergruppen. Im früheren sozialistischen Raum waren Besitz- und Zugangsrechte sowie Zuständigkeiten des Managements in der Regel eindeu- tig definiert. Dieses hierarchisch aufgebaute zentralistische institutionelle Gefüge, das zuvor autochthone kommunale oder religiöse Institutionen eliminierte, kollabierte mit dem Ende des sozialistischen Experiments. Somit führte der Wegfall dieses dominanten Institutionenkörpers in postsozialistischen Gesellschaften zu institutionellen Lücken, Verunsicherung und Marginalisierung. Mit struktureller Macht ausgestattete Akteure, wie ehemalige Parteikader oder Kolchosleiter, profitierten oftmals von diesen institutionellen Lücken und sicherten sich die wertvollsten Ressourcen, was einfachen Arbeitern kaum möglich war. Der Fokus muss somit auf einer Analyse akteursspezifischer Verfügungsrechte an Naturressourcen und den unterschiedlichen Handlungsoptionen liegen. Eine solche Betrachtung schließt auch die unterschiedliche Ressourcenausstattung von Frauen und Männern sowie geschlechtsspezifische Repräsentanz in Institutionen ein. Die unterschiedlichen produktiven und reproduktiven Aufgaben in Haushalt und Gesellschaft führen zu verschiedenen Zugängen zu und Verantwortlichkeiten gegenüber Ressourcen, aber auch zu einer differenten Wahrnehmung der Umwelt und einem unterschiedlichen „Geographie machen“. Die geschlechterunabhängige Förderung von Bildung, das Aufbrechen genderspezifischer Traditionen und die Einbindung von Frauen in außerhäusliche Wirtschaftsbereiche war sicherlich eine der größten emanzipatorischen Leistungen des Sozialismus. Dennoch muss hier gefragt werden, wie diese Prozesse einerseits zu erhöhter Belastung der Betroffenen und zu gesellschaftlicher Emanzipation von Frauen führten und wie sich andererseits diese Rollenmuster in der postsozialistischen Zeit durchgepaust oder gewandelt haben.

\subsection{Differenzierter Blick auf Akteure und regionale Zusammenhänge}

"Someone's degradation is someone else's accumulation" (Dove und Hudayana, 2008) - Ressourcenentnahme und Umwelteingriffe sind für die einen mit Profit, für andere mit Schaden verbunden. Diese schlichte Wahrheit muss bei der Analyse von Mensch-Umwelt-Verhältnissen stets berücksichtigt werden. Es gibt selten ein offensichtliches ,richtig“ oder ,falsch“, sondern viel eher unterschiedliche Optionen, von denen eine Seite profitiert, eine andere nicht. Nur eine Ausdifferenzierung oftmals pauschal geäußerter Kritik an Neoliberalisierung, Globalisierung oder am Sozialismus trägt zu einer konzeptionellen Weiterentwicklung der Politischen Ökologie bei.

Ebenfalls muss die zeitliche Dimension Berücksichtigung finden, denn Eingriffe in den Naturhaushalt oder Ressourcennutzungen sind möglicherweise mit kurzfristigen Gewinnen, aber langfristigen Verlusten verbunden. Bewertungen von anthropogenen Umweltaktivitäten müssen somit immer eine differenzierte Analyse der verschiedenen Akteure und deren Interessen sowie der kurz- und langfristigen ökologischen, ökonomischen oder gesellschaftlichen Folgen bein- 
halten. Auch die Zusammensetzung der Akteursgruppen und deren Intentionen verändern sich oftmals und bedürfen stets neuer Analyse. Gerade historische Zäsuren wie in den postsozialistischen Regionen führen zu Neupositionierungen von Akteuren und Verlagerungen ihrer Interessen.

Politisch-ökologische Studien basieren oft auf empirischen Untersuchungen in lokalen Kontexten. Dieses ideographische Vorgehen erscheint notwendig, um die Komplexität und Konfigurationen von Strukturen, Prozessen und Handlungen zu verstehen und eine substantielle soziopolitische und historische Tiefe zu erreichen. Erforderlich ist allerdings auch, die lokale Ebene mit anderen räumlichen Ebenen in Verbindung zu setzen, um die „localist trap“ (Muldavin, 2008:692) zu vermeiden und im Sinne induktiven Vorgehens Verallgemeinerungen abzuleiten. Individuen, Orte und Regionen sind im Zuge einer verdichteten Globalisierung durch Flüsse von Wissen, Kapital, Gütern und Menschen verbunden. Selbst periphere Gemeinschaften sind in globale Prozesse und Netze eingebunden. Doch zu einer Angleichung, einer Konvergenz zwischen postsozialistischen Gesellschaften und dem Westen oder Entwicklungsländern im Globalen Süden führt dies nicht notwendigerweise. Das Modell der „fragmentierenden Entwicklung“ von Scholz (2004) scheint hier eher angebracht. Demnach können einzelne Regionen und Gruppen die Chancen der Globalisierung nutzen, während ein Großteil möglicherweise zunehmend marginalisiert wird. Deswegen muss die Betrachtung einer bloßen Dualität zwischen West und Ost, wie sie zu Zeiten des Kalten Krieges angewandt wurde, oder auch die Nutzung anderer Dichotomien vermieden werden, müssen vielmehr gesellschaftliche Kontexte, regionale und lokale Besonderheiten, kurzum die Embeddedess berücksichtigt werden.

\section{Fazit}

Die gegenwärtigen postsozialistischen Regionen und Gesellschaften sind durch vielfältige Transformationen wie externe oder koloniale Interventionen, sozialistische Revolutionen und die Auflösung des Staatssozialismus geprägt, womit die Relevanz historischer Analysen untermauert wird. Eine Politische Ökologie des Postsozialismus sollte darauf zielen, Verständnis über die Gesellschaft-Umwelt-Verhältnisse im Sozialismus und im Postsozialismus zu gewinnen, auf Basis empirischer Erhebungen Darstellungen und Erklärungen zu den politischen Einflüssen auf Natur- und Umweltschutz, Ressourcenmanagement und Umweltwandel zu liefern, um Ansatzpunkte für gesellschaftlich gerechtere und umweltdienlichere, nachhaltige Entwicklungsmöglichkeiten aufzuzeigen. In der postsozialistischen Sphäre haben sich durch umfassende Transformationsprozesse, die Erosion bestehender Sicherheitsmechanismen, die Neukonfiguration von Institutionen sowie Globalisierungseinflüsse die Gesellschaft-Umwelt-Verhältnisse grundlegend transformiert und somit auch die Position von Akteuren sowie die
Bedeutung von Natur und Naturressourcen. Gleichzeitig persistieren zu einem nicht unbedeutenden Grad institutionelle Strukturen und Umweltperzeptionen aus der Zeit des real existierenden Sozialismus und sind die sozialistischen Materialitäten in Form von Siedlungen, Infrastrukturen oder Agrarlandschaften als socialist legacies bis heute prägend. Eine erhöhte Nachfrage nach knappen Ressourcen, zunehmende Konkurrenzen und beschleunigter Umweltwandel tragen zu einer weiteren „Politisierung der Umwelt“ (Krings und Müller, 2001) bei. Eine Politische Ökologie des Postsozialismus erscheint notwendig. Denn Gesellschaft und Natur gemeinsam zu denken, sich gleichzeitig mit sozialer Gerechtigkeit und Naturschutz sowie mit den alltäglichen Herausforderungen und Nöten Betroffener zu beschäftigen, bleibt aktuell.

Edited by: A. Otto

Reviewed by: two anonymous referees

\section{Literatur}

Adger, W. N., Benjaminsen, T. A., Brown, K., und Svarstad, H.: Advancing a political ecology of global environmental discourses, Dev. Change, 32, 681-715, 2001.

Berkes, F.: Sacred Ecology: Traditional Ecological Knowledge and Resource Management, Taylor and Francis, Philadelphia, 1999.

Bertelsmann Stiftung (Hrsg.): Transformation Index BTI 2016 Political Management in International Comparison, Bertelsmann Stiftung, Gütersloh, 2016.

Blaikie, P.: The Political Economy of Soil erosion in Developing Countries, Longman, New York, 1985.

Blaikie, P. und Brookfield, H.: Land Degradation and Society, Methuen, London, 1987.

Blomley, N.: The territory of property, Prog. Hum. Geog., 30, doi:10.1177/0309132515596380, 2015.

Bolotova, A.: Die Geologen: Kolonisatoren am Lagerfeuer. Selbstbild und Naturverständnis in der Sowjetunion, Osteuropa, 58, 57-67, 2008.

Botkin, D. B.: Discordant Harmonies: a New ecology for the Twenty-first Century, Oxford University Press, Oxford, 1990.

Bridge, G.: Natural resources, in: International Encyclopedia of Human Geography. Elsevier, Warwick, 261-268, 2009.

Bridge, G., McCarthy, J., und Perreault, T.: Editors' introduction, in: The Routledge Handbook of Political Ecology, Herausgeber: Perreault, T., Bridge, G., und McCarthy, J., Routledge, Abingdon, 3-18, 2015.

Bryant, R. L.: Political ecology: an emerging research agenda in Third-World studies, Polit. Geogr., 11, 12-36, 1992.

Bryant, R. L. (Hrsg.): The International Handbook of Political Ecology, Elgar, Cheltenham, 2015.

Bryant, R. L. und Bailey, S.: Third World Political Ecology, Routledge, London, 1997.

Bryant, R. L., Paniagua, A., und Kizos, T.: Conceptualising "shadow landscape" in political ecology and rural studies, Land Use Policy, 28, 460-471, 2011.

Castree, N.: Capitalism and the Marxist critique of political ecology, in: The Routledge Handbook of Political Ecology, Herausgeber: 
Perreault, T., Bridge, G., und McCarthy, J., Routledge, Abingdon, 279-292, 2015.

Davis, D. K.: Historical political ecology: on the importance of looking back to move forward, Geoforum, 40, 285-286, 2009.

Dörre, A.: Naturressourcennutzung im Kontext struktureller Unsicherheiten. Eine Politische Ökologie der Weideländer Kirgisistans in Zeiten gesellschaftlicher Umbrüche, Erdkundliches Wissen, Band 154, 416 pp., Steiner, Stuttgart, 2014.

Dörre, A. und Schmidt, M.: Vom Schutz und Nutzen von Wäldern: Kirgistans Nusswälder im Lichte historischer und aktueller Schutzdiskurse, Geogr. Z., 96, 207-227, 2008.

Dove, M. R. und Hudayana, B.: The view from the volcano: an appreciation of the work of Piers Blaikie, Geoforum, 39, 736-746, 2008.

Elmhirst, R.: Introducing new feminist political ecologies, Geoforum, 42, 129-132, 2011.

Engel-Di Mauro, S.: Seeing the local in the global: political ecologies, world-systems, and the question of scale, Geoforum, 40, 116-125, 2009.

Escobar, A.: Encountering Development: the Making and Unmaking of the Third World, Princeton University Press, Princeton, 1995.

Escobar, A.: Constructing nature: elements for a poststructural political ecology, in: Liberation Ecologies: Environment, Development, Social Movements, Herausgeber: Peet, R. und Watts, M., Routledge, London, 46-68, 1996.

Féaux de la Croix, J.: Erfahrungen mit den sowjetischen und postsowjetischen Staudammprojekten am Naryn Fluss, in: Aktuelle Forschungen zu den Mensch-Umwelt-Verhältnissen in Kirgistan, Herausgeber: Schmidt, M., Hannoversche Geographische Arbeiten, 62, Lit, Münster, 90-100, 2014.

Flitner, M.: Lärm an der Grenze. Fluglärm und Umweltgerechtigkeit am Beispiel des Flughafens Basel-Mulhouse, Erdkundliches Wissen, Band 140, 238 pp., Steiner, Stuttgart, 2007.

Forsyth, T.: Critical Political Ecology: the Politics of Environmental Science, Routledge, London, 2003.

Freedom House (Hrsg.): Freedom in the World 2015: Discarding Democracy: Return to the Iron Fist, Washington DC, 2015.

Hajer, M. A.: The Politics of Environmental Discourse, Clarendon, Oxford, 1995.

Hann, C. (Hrsg.): Postsozialismus: Transformationsprozesse in Europa und Asien aus ethnologischer Perspektive, Campus, Frankfurt, 2002

Haraway, D.: Situated knowledges: the science question in feminism and the privilege of partial perspective, Feminist Stud., 14, 575-599, 1988.

Harper, K.: Wild Capitalism: Environmental Activists and Postsocialist Political Ecology in Hungary, Columbia University Press, New York, 2006.

Harré, R., Brockmeier, J., und Mühlhäusler, P.: Greenspeak: a Study of Environmental Discourse, Sage, Thousand Oaks, 1999.

Hartwig, J.: Die Vermarktung der Taiga: Die Politische Ökologie der Nutzung von Nicht-Holz-Waldprodukten und Bodenschätzen in der Mongolei, Erdkundliches Wissen, Band 143, 436 pp., Steiner, Stuttgart, 2007.

Hobsbawm, E.: Das Zeitalter der Extreme: Weltgeschichte des 20. Jahrhunderts, Hanser, München, 1995.

Humphrey, C.: Ist "postsozialistisch" noch eine brauchbare Kategorie? in: Postsozialismus: Transformationsprozesse in Europa und Asien aus ethnologischer Perspektive, Herausgeber: Hann, C., Campus, Frankfurt, 26-31, 2002.

Kornai, J.: Economics of Shortage, North-Holland Publisher, Amsterdam, 1980.

Krings, T.: Politische Ökologie: Grundlagen und Arbeitsfelder eines geographischen Ansatzes der Mensch-Umwelt-Forschung, Geographische Rundschau, 60, 4-9, 2008.

Krings, T.: Politische Ökologie, in: Geographie: Physische Geographie und Humangeographie, Herausgegeber: Gebhardt, H., Glaser, R., Radtke, U., und Reuber, P., Spektrum, München, $1097-$ 1106, 2011.

Krings, T. und Müller, B.: Politische Ökologie: theoretische Leitlinien und aktuelle Forschungsfelder, in: Politische Geographie: handlungsorientierte Ansätze und Critical Geopolitics, Herausgegeber: Reuber, P. und Wolkersdorfer, G., Spektrum, Heidelberg, 93-116, 2001.

Lindner, P.: Der Kolchoz-Archipel im Privatisierungsprozess: Wege und Umwege der russischen Landwirtschaft in die globale Marktgesellschaft, Transcript, Bielefeld, 2008.

Loomba, A.: Colonialism/Postcolonialism, Routledge, London, 2005.

Marat, E.: National Ideology and State-building in Kyrgyzstan and Tajikistan, Central Asia-Caucasus Institute, Washington, 2008.

Marston, S. A.: The social construction of scale, Prog. Hum. Geog., 24, 219-242, 2000.

McCarthy, J.: First World political ecology: lessons from the Wise Use Movement, Environ. Plann. A, 34, 1281-1302, 2002.

McCay, B. J. und Acheson, J. M.: The Question of the Commons: the Culture and Ecology of Communal Resources, University of Arizona Press, Tucson, 1987.

Meessen, H.: Anspruch und Wirklichkeit von Naturschutz und Landschaftspflege in der Sowjetunion, AG Geographica Bernensia, Bern, 1992.

Moore, P. D., Chaloner, W. G., und Stott, P.: Global Environmental Change, Blackwell Science, Oxford, 1996.

Muldavin, J.: Assessing environmental degradation in contemporary China's hybrid economy: state policy reform and agrarian dynamics in Heilongjiang province, Ann. Assoc. Am. Geogr., 87, 579-613, 1997.

Muldavin, J.: The time and place for political ecology: an introduction to the articles honouring the life-work of Piers Blaikie, Geoforum, 39, 687-697, 2008.

Nash, C.: Cultural geography: postcolonial cultural geographies, Prog. Hum. Geog., 26, 219-230, 2002.

Neumann, R. P.: Making Political Ecology, Hodder Arnold, New York, 2005.

Neumann, R. P.: Political ecology: theorizing scale, Prog. Hum. Geog., 33, 398-406, 2009.

Nove, A.: Das sowjetische Wirtschaftssystem, Nomos, BadenBaden, 1980.

Parsons, T.: Das System moderner Gesellschaften, Juventa, München, 1972

Peet, R. und Watts, M. (Hrsg.): Liberation Ecologies: Environment, Development, Social movements, Routledge, London, 1996.

Peet, R., Robbins, P., und Watts, M. (Hrsg.): Global Political Ecology, Routledge, London, 2011.

Perreault, T., Bridge, G., und McCarthy, J. (Hrsg.): The Routledge Handbook of Political Ecology, Routledge, Abingdon, 2015. 
Robbins, P.: Political Ecology: a Critical Introduction, Blackwell, Malden, 2012.

Rocheleau, D., Thomas-Slayter, B., und Wangari, E. (Hrsg.): Feminist Political Ecology: Global Issues and Local Experiences, Routledge, London, 1996.

Russische Akademie der Wissenschaften (Hrsg.): Rossijskij srednij klass v uslovijach stabil'nosti i krizisov (Die russische Mittelschicht in Zeiten von Stabilität und Krise), Institut für Soziologie, Moskau, 2016.

Sayre, N. F.: Ecological and geographical scale: parallels and potential for integration, Prog. Hum. Geog., 29, 276-290, 2005.

Schmidt, M.: Die Erfindung Kirgistans und der unvollendete Prozess der Nationenbildung, Europa Regional, 15, 209-223, 2007.

Schmidt, M.: Mensch und Umwelt in Kirgistan: Politische Ökologie im postkolonialen und postsozialistischen Kontext, Erdkundliches Wissen, 153, Steiner, Stuttgart, 2013.

Schmidt, M. und Pearson, O.: Pastoral livelihoods under pressure: ecological, political and socioeconomic transitions in Afar (Ethiopia), J. Arid Environ., 124, 22-30, 2016.

Schneider, B.: In den Tiefen des Tropenwaldes. Eine politischökologische Betrachtung des Gold- und Diamantenbergbaus in Venezuela, Ibidem, Stuttgart, 2002.

Scholz, F.: Geographische Entwicklungsforschung: Methoden und Theorien, Gebrüder Borntraeger, Stuttgart, 2004.

Smith, N.: Uneven Development: Nature, Capital, and the Production of Space, Blackwell, Oxford, 1984.
Soliva, R.: Der Naturschutz in Nepal: eine akteursorientierte Untersuchung aus der Sicht der Politischen Ökologie, LIT, Münster, 2002.

Staddon, C.: Towards a critical political ecology of human-forest interactions: collecting herbs and mushrooms in a Bulgarian locality, T. I. Brit. Geogr., NS34, 161-176, 2009.

Steimann, B.: Making a Living in Uncertainty: Agro-pastoral Livelihoods and Institutional Transformations in Post-socialist Rural Kyrgyzstan, Department of Geography, Zürich, 2011.

Swyngedouw, E. und Heynen, N. C.: Urban political ecology, justice and the politics of scale, Antipode, 35, 898-918, 2003.

UNDP: Human Development Report 2015: Work for Human Development, UNDP, New York, 2015.

Visser, O. und Spoor, M.: Land grabbing in post-Soviet Eurasia: the world's largest agricultural land reserves at stake, J. Peasant Stud., 38, 299-323, 2011.

Watts, M.: Silent Violence: Food, Famine and Peasantry in Northern Nigeria, University of California Press, Berkeley, 1983.

Watts, M.: Review of Land Degradation and Society by Piers Blaikie and Harold Brookfield, Capitalism Nature Socialism, 1, 123131, 1990.

World Bank: World Development Report: From plan to market, Washington DC, 1996.

Zimmerer, K. S. und Bassett, T. J.: Political Ecology: an Integrative Approach to Geography and Environment-development Studies, Guilford, New York, 2003. 